\title{
Electrochemical behaviour of superhydrophobic coating fabricated by spraying a carbon nanotube suspension
}

\author{
L BELSANTI $^{1, *}$, H OGIHARA ${ }^{2}$, S MAHANTY ${ }^{3}$ and G LUCIANO ${ }^{1}$ \\ ${ }^{1}$ CNR-ISMAR, Istituto di Scienze Marine, Genova 16149, Italy \\ ${ }^{2}$ Department of Chemistry and Materials Science, Tokyo Institute of Technology, Tokyo 152-8552, Japan \\ ${ }^{3}$ Indian Institute of Technology Kanpur, Kanpur 208016, India
}

MS received 30 June 2014; revised 12 September 2014

\begin{abstract}
In this study, superhydrophobic films were prepared through a spraying process of carbon nanotube (CNT) suspension on aluminium alloy substrate and characterized by scanning electronic microscope (SEM) contact angles (CAs) and potentiodynamic polarization tests in $0.1 \mathrm{M} \mathrm{NaCl}$ solution. Results indicate the positive effect of superhydrophobic film on the $\mathrm{CA}\left(>160^{\circ}\right)$ in comparison with the bare metal due to the high porosity density at the surface caused by the presence of CNTs. The electrochemical observations indicate the presence of a positive shift of $E_{\text {corr }}$ that confers a better corrosion resistance of the coated samples.
\end{abstract}

Keywords. Al alloy; surface coating; superhydrophobicity; potentiodynamic polarization.

\section{Introduction}

Aluminium and its alloys exhibit high-specific strength, low density, excellent heat and electric conductivities and lowspecific weight. ${ }^{1,2}$ These combinations of attractive properties make it useful in household and industrial fields such as marine, automotive and aerospace industries. In contrast the presence of precipitates in the $\mathrm{Al}$ alloy turns it to pitting susceptibility in the chloride/marine environment.

The presence of a spontaneously formed thin compact oxide film on the metal surface makes aluminium highly resistant to corrosion. However, aluminium is active and prone to corrosion in contact with water, especially in corrosive environment such as in aqueous solution containing $\mathrm{Cl}^{-}$ions, which restricts the application of aluminium and its alloy in many engineering fields. ${ }^{3}$ Many studies have been conducted to improve the corrosion resistance of aluminium and its alloy in corrosion environment, in order to extend their applications in marine engineering field.

Attention to superhydrophobic surfaces has been growing over recent decades. Especially, superhydrophobic surfaces with contact angle (CA) greater than $150^{\circ}$ have attracted much interest for their applications due to their self-cleaning and wettability properties. ${ }^{4,5}$ These surfaces are of special interest, in fact, due to their anti-sticking, anti-contamination and self-cleaning properties.

Two different approaches have been till now developed for the fabrications of artificial superhydrophobic surfaces: surface roughing of hydrophobic materials or chemical modification of rough surface by low surface energy materials. ${ }^{6,7}$ Traditionally, superhydrophobic metallic surfaces

\footnotetext{
*Author for correspondence (lucrezia.belsanti@ge.ismar.cnr.it)
}

were obtained combining micro/nanostructures with low surface energy materials.

Many methods and techniques have been developed to create rough surfaces with different microstructures, such as electrochemical deposition, ${ }^{8}$ anodization, ${ }^{9}$ phase separation, ${ }^{10}$ sol-gel process, ${ }^{11}$ layer-by-layer assembly ${ }^{12,13}$ and chemical etching method. ${ }^{14}$

The characteristics of water repellency and low adhesion makes superhydrophobic surface suitable to improve anticorrosion performances of various engineering material surfaces, such as steel, copper, zinc, titanium and magnesium. In fact, these superhydrophobic treatments also contribute to an effective barrier to keep water, moisture and corrosive medium away from contacting and reacting with the metal. ${ }^{15,16}$

A rapid method to create superhydrophobic surfaces on magnesium alloy has been developed by Ishizaki et al, ${ }^{17,18}$ using cerium oxide modified microstructure film with fluoroalkylsilane. It has been reported that the superhydrophobic treatment makes magnesium alloy highly corrosion resistant

Hu et $a l^{19}$ observed CA (with water) $>170^{\circ}$ for superhydrophobic $\mathrm{TiO}_{2}$ film on the Hastelloy substrate through fluoroalkylsilane molecules modification. Superhydrophobic films on titanium CA (with water) $>160^{\circ}$ were fabricated by Zhang $e t a l^{20}$ through the combination of electrochemical oxidation and later by perfluorooctyl triethoxysilane modification, these films also show effective anti-corrosion properties.

Su $\mathrm{et}_{\mathrm{al}} \mathrm{l}^{16}$ obtained hydroxide zinc carbonate film on zinc plates, their surface wettability of the plates move from superhydrophilic to superhydrophobic through the solvent alternation. The electrochemical behaviour of these surfaces was not characterized. 
Recently, we have reported facile methods to fabricate superhydrophobic films by spraying nanomaterials such as hydrophobic silica nanoparticles and carbon nanotubes (CNTs). ${ }^{21,22}$ The feature of these spray-coating technique are its simplicity, more cost effectiveness and less complex condition to prepare the superhydrophobic coatings.

In the present study, we examined the corrosion resistance of the spray-coated superhydrophobic films on aluminium alloy substrates, in order to explore their suitability as anti-corrosion barrier in marine environment. ${ }^{23,24}$

\section{Experimental}

\subsection{Materials}

Carbon nanotubes (CNT; Showa Denko Co., average diameter $\sim 150 \mathrm{~nm}$; length $\sim 10-20 \mu \mathrm{m}$ ), trimethylsiloxysilicate (TMSS, SR1000; Momentive performance materials) and ethanol (99.5\%, Kanto Kagaku Co., Ltd) were used without further purification.

Aluminium plates of commercial Al 6082 and Al5754H111 were selected, as both these alloys are used in marine environment due to their anti-corrosion properties.

A $0.1 \mathrm{M}$ solution of $\mathrm{NaCl}$ in water was used as electrolyte for the potentiodynamic measurements.

\subsection{Preparation of superhydrophobic coatings}

Superhydrophobic coatings were prepared as reported by Ogihara et al. ${ }^{22}$

CNT $(0.3 \mathrm{~g})$ and TMSS $(0.15 \mathrm{~g})$ were added to $30 \mathrm{ml}$ of ethanol, and the solution was stirred and sonicated to form suspension. The suspension was carefully sprayed over substrates (aluminium plates) in order to form a complete coverage over the surface of the samples. The resulting samples were placed horizontally and dried at room temperature.

\subsection{Characterization}

The CA of water droplets $(5 \mu \mathrm{l})$ on the superhydrophobic coating was measured using a CA metre (OCA 15 plus dataphysics). The average water $\mathrm{CA}$ value was obtained by sampling five areas on the surface of the samples.

The surface morphologies of the obtained samples were investigated by scanning electron microscope (LEO 1450 VPScanning Electron Microscope) at an acceleration voltage of $20 \mathrm{kV}$. Microanalysis was performed at $20 \mathrm{kV}$, the working distance was $15 \mathrm{~mm}$ and the chamber vacuum was $6.66 \times 10^{-4} \mathrm{~Pa}$. Images were obtained with secondary electrons mode.

Potentodynamic polarization tests were carried out by a PGSTAT 30 Autolab instrument (Metrohm) in $0.1 \mathrm{M}$ solution of $\mathrm{NaCl}$ in water at room temperature with a three-electrode system. It contains working electrode, counter electrode and reference electrode. Saturated calomel electrode was used as a reference electrode, whereas platinum plate was used as a counter electrode. All tests were conducted at ambient temperature at a scanning rate of $10 \mathrm{mV} \mathrm{s}^{-1}$ from -1100 to $+1000 \mathrm{mV}$. All measurements were performed with respect to the reference electrode.

\section{Results and discussion}

\subsection{Morphology and wettability of sample}

Figure 1 shows SEM images of superhydrophobic coating over Al alloy substrate (Al 6082). The components of the coating are CNTs and TMSS, both of which display hydrophobic properties. As TMSS in the coating acts like glue that connects CNTs, ${ }^{22}$ the CNT/TMSS composite coatings are expected to feature mechanical strength. It can be observed (figure 1) that CNTs were randomly stacked and created a large amount of porous structure on the surface. It is well known that the presence of air in the porous structure amplifies hydrophobicity of surface, which results in exhibiting superhydrophobicity.

Figure 2 shows SEM images taken on the border of bare and coated Al alloy substrate (Al 6082). The coated part of the substrates shows a uniform morphology on the surface of the samples. Uniformity of superhydrophobic coating is an important requirement in order to use the coating as an anti-corrosion barrier.

CA for the measured samples is $160^{\circ}$ (figure 3), in accordance with the results obtained by spraying the samples on glass cover substrates. ${ }^{22}$

\subsection{Electrochemical analysis}

Potentiodynamic polarization curves of uncoated samples (Al 5754-H111 and Al 6082) and coated Al alloy substrates (Al 6082) are shown in figure 4.

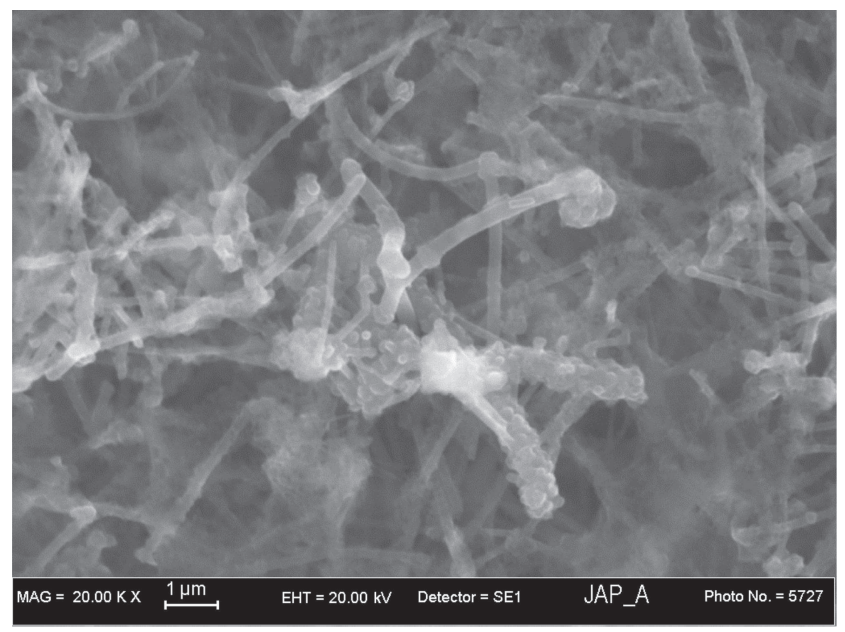

Figure 1. SEM image of carbon nanotube/TMSS composite superhydrophobic coating over Al alloy (Al 6082). The Al alloy substrate in not visible because it is completely covered by superhydrophobic coating. 


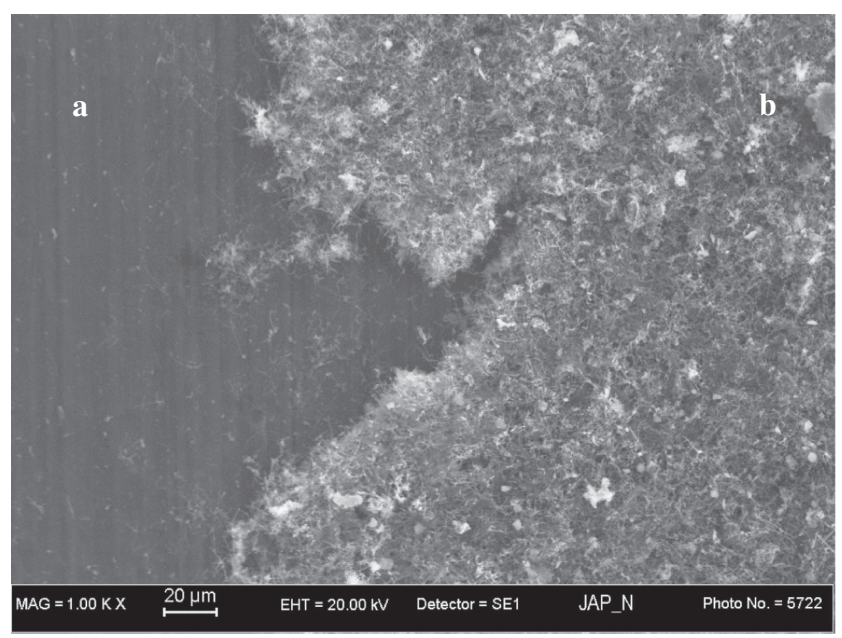

Figure 2. SEM image on the border of bare and coated $\mathrm{Al}$ alloy (Al 6082): (a) Al alloy substrate ( $\mathrm{Al}$ 6082) and (b) carbon nanotube/ TMSS composite coating.

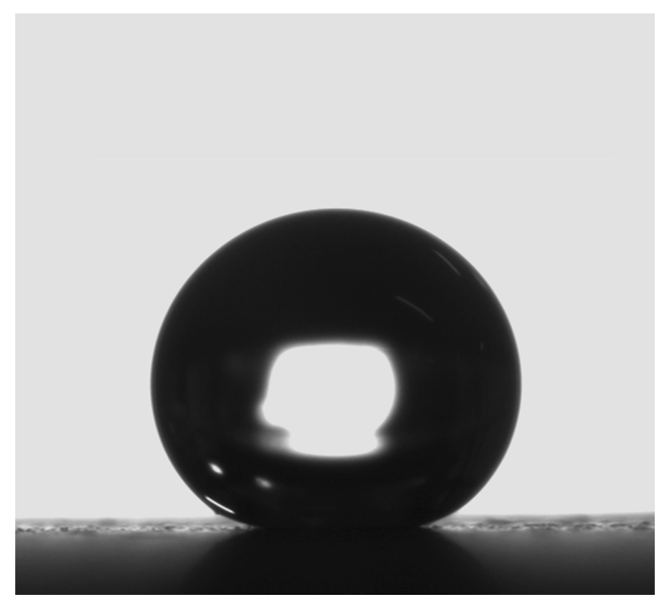

Figure 3. Measured CA of $160^{\circ}$ on superhydrophobic sample.

It can be clearly seen that the corrosion potential ( $\left.E_{\text {corr }}\right)$ of the superhydrophobic surface was fairly positive compared with uncoated $\mathrm{Al}$ alloy samples. Results indicate that the pre- and post-coated samples were anodically active. Shift in $E_{\text {corr }}$ towards higher positive potential indicates that the coated surface is more corrosion resistant as compared to $\mathrm{Al}$ alloy samples (Al 5754-H111 and $\mathrm{Al}$ 6082). Figure 5 shows the variation of $E_{\text {corr }}$ potential with the bare $\mathrm{Al}$ alloy samples (Al 5754-H111 and Al 6082) and coated surface. There is a 1.5 and 1.25 time decrement of $E_{\text {corr }}$ value of coated sample compared to bare Al 5754-H111 and Al 6082, clearly indicating the positive effect in corrosion activity. The breakdown potential $(-0.695 \mathrm{~V})$ is also observed in $\mathrm{Al} 6082$ sample, clearly indicating the pitting activity of $\mathrm{Al}$ alloy, whereas coated sample and Al 5754-H111 did not show any pitting activity in the chlorine medium. The $E_{\text {corr }}$ and $I_{\text {corr }}$ (corrosion current), characteristics of coated sample show less increment of corrosion current with potential in anodic

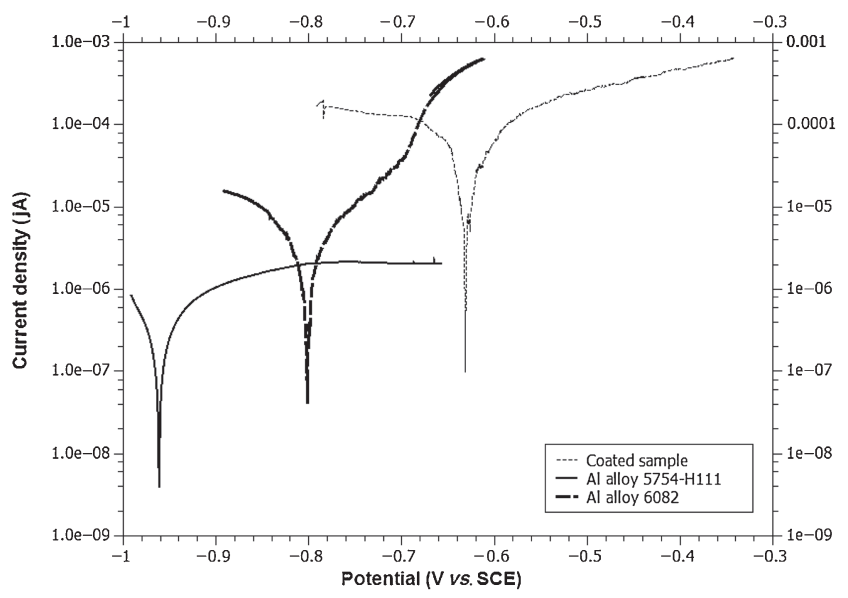

Figure 4. Potentiodynamic curves for uncoated samples (Al 5754-H111 and Al 6082) and coated surface (superhydrophobic coating over Al 6082).

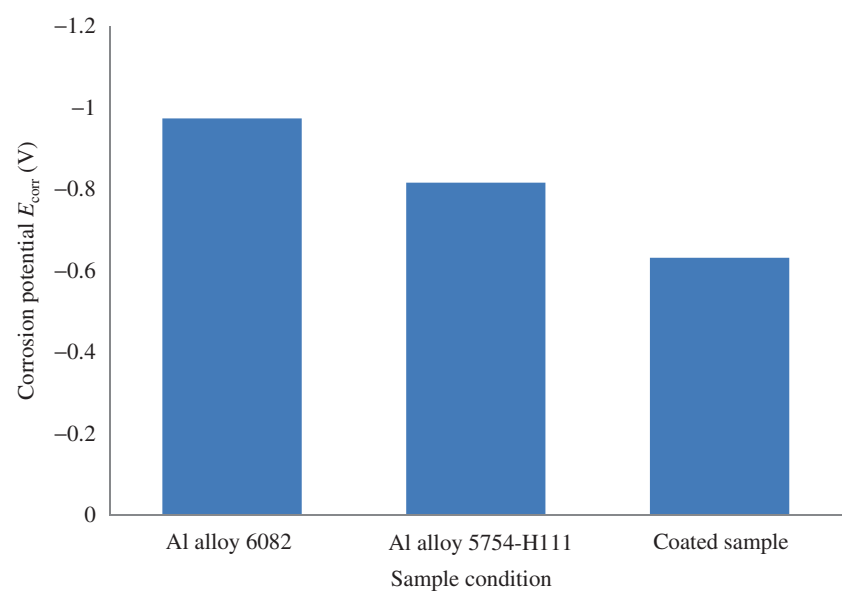

Figure 5. Variation of $E_{\text {corr }}$ potential for uncoated samples (Al 5754-H111 and Al 6082) and coated surface (superhydrophobic coating over Al 6082).

region as compared to Al 6082 sample. The observation shows a higher passivity of coated sample in comparison with $\mathrm{Al} 6082$ sample. However, the $I_{\text {corr }}$ value of coated surface was higher than Al 5754-H111 and Al 6082 samples. The improvement of the corrosion resistance of superhydrophobic coating is based on the fact that the coated layer prevents the $\mathrm{Al}$ alloy surface to expose in the chloride medium, whereas $\mathrm{Al}$ alloy surface exposed to chloride medium undergoes corrosion attack due to the presence of precipitate, which acts like cathode and $\mathrm{Al}$ matrix as anode. The exposure in the chloride medium is prevented by the coating and thus improves the corrosion resistivity.

\section{Conclusions}

In this study, superhydrophobic coatings were successfully fabricated on $\mathrm{Al}$ alloys through spray coating. The analysis revealed the increment in the hydrophobicity nature of bare 
$\mathrm{Al}$ alloy samples (Al 5754-H111 and Al 6082). The investigation drew attention to remarkable improvement of the corrosion potential with superhydrophobic coatings in $0.1 \mathrm{M}$ solution of $\mathrm{NaCl}$. The corrosion potential sequence for $\mathrm{Al}$ $6082: \mathrm{Al} 5754-\mathrm{H} 111:$ coated $\mathrm{Al}$ alloy is $-0.94:-0.82:-0.65$ $\mathrm{V}$, respectively. The coated sample is also highly resistive to the pitting corrosion compared to the bare $\mathrm{Al} 6082$ alloy. The significant improvement in the corrosion resistance properties has been achieved after superhydrophobic coating, which can be related to good adhesion, metallurgical bond and passive film between the coating and the substrate.

The advantages of these spray-coating techniques related to its low cost, easy conditions of synthesis and application, and anti-corrosion barrier make the coating suitable for further study for its application in marine environment.

\section{Acknowledgements}

This study is supported by a research grant from The Mazda Foundation and has been partially funded by the Flagship Project RITMARE-The Italian Research for the Seacoordinated by the Italian National Research Council and funded by the Italian Ministry of Education, University and Research within the National Research Program 2011-2013. We would like to thank Dr Giuliano Greco for providing useful suggestions on the writing of the present paper.

\section{References}

1. Abdel-Gaber A M, Abd-El-Nabey B A, Sidahmed I M, ElZayady A M and Saadawy M 2006 Mater. Chem. Phys. 98 291

2. Yin B, Fang L, Tang A Q et al 2011 Appl. Surf. Sci. 258580
3. Badawy W A, Al-Kharafi F M and El-Azab A S 1999 Corros. Sci. 41709

4. Feng X and Jiang L 2006 Adv. Mater. 183063

5. Roach P, Shirtcliffe N J and Newton M I 2008 Soft Matter 4 224

6. Burkarter E, Saul C K, Thomazi F et al 2007 J. Phys. D: Appl. Phys. $\mathbf{4 0} 7778$

7. Meng L Y and Park S J 2010 J. Colloid Interface Sci. 342559

8. Li M, Zhai J, Liu H, Song Y, Jiang L and Zhu D 2003 J. Phys. Chem. B 1079954

9. Zhang L, Zhou Z, Cheng B, DeSimone J M and Samulski E T 2006 Langmuir 228576

10. Nakajima A, Abe K, Hashimoto K and Watanabe T 2000 Thin Solid Films 376140

11. Venkateswara Rao A, Latthe S S, Nadargi D Y, Hirashima H and Ganesan V 2009 J. Colloid Interface Sci. 332484

12. Zhao N, Shi F, Wang Z and Zhang X 2005 Langmuir 214713

13. Tsai H J and Lee Y L 2007 Langmuir 2312687

14. Qian B and Shen Z 2005 Langmuir 219007

15. Liang J, Hu Y, Wu Y and Chen H 2013 J. Nanomater. 20136

16. Su B, Li M, Shi Z and Lu Q 2009 Langmuir 253640

17. Ishizaki T and Saito N 2010 Langmuir 269749

18. Ishizaki T, Masuda Y and Sakamoto M 2011 Langmuir 27 4780

19. Hu Y W, Huang S Y, Liu S et al 2012 Appl. Surf. Sci. 2587460

20. Zhang F, Chen S, Dong L, Lei Y, Liu T and Yin Y 2011 Appl. Surf. Sci. 2572587

21. Ogihara H, Xie J, Okagaki J and Saji T 2012 Langmuir 28 4605

22. Ogihara H, Xie J and Saji T 2014 J. Mater. Sci. 493183

23. Li X M, Reinhoudt D and Crego-Calama M 2007 Chem. Soc. Rev. 361350

24. Ogihara H, Okagaki J and Saji T 2011 Langmuir 279069 